Documentation et bibliothèques

DOCUMENTATION BIBLIOTHEQUES

\title{
Les bibliothèques à double allégeance : évolution du concept
}

\section{Réal Messier}

Volume 23, numéro 4, décembre 1977

URI : https://id.erudit.org/iderudit/1055207ar

DOI : https://doi.org/10.7202/1055207ar

Aller au sommaire du numéro

\section{Éditeur(s)}

Association pour l'avancement des sciences et des techniques de la documentation (ASTED)

\section{ISSN}

0315-2340 (imprimé)

2291-8949 (numérique)

Découvrir la revue

\section{Citer cet article}

Messier, R. (1977). Les bibliothèques à double allégeance : évolution du concept. Documentation et bibliothèques, 23(4), 197-201.

https://doi.org/10.7202/1055207ar

\section{Résumé de l'article}

Le concept de l'intégration des bibliothèques publique et scolaire n'est pas nouveau : au siècle dernier, les premières bibliothèques publiques étaient à peine apparues aux États-Unis qu'on leur confiait déjà une mission éducative. Certaines expériences entreprises au Québec furent sans lendemain parce qu'il s'agissait beaucoup plus de juxtaposition que d'intégration des services. L'idée de l'intégration des deux réseaux, reprise récemment par le gouvernement du Québec, se heurte à deux réalités : la très grande différence des milieux à desservir (scolaire et public) et l'évolution même des bibliothèques publiques qui deviendront sans doute davantage des services d'information que des agents d'éducation.
Tous droits réservés (C) Association pour l'avancement des sciences et des techniques de la documentation (ASTED), 1977
Ce document est protégé par la loi sur le droit d'auteur. L’utilisation des services d'Érudit (y compris la reproduction) est assujettie à sa politique d'utilisation que vous pouvez consulter en ligne.

https://apropos.erudit.org/fr/usagers/politique-dutilisation/ 


\title{
Les bibliothèques à double allégeance: évolution du concept
}

\author{
Réal Messier \\ École de bibliothéconomie \\ Université de Montréal
}

Le concept de l'intégration des bibliothèques publique et scolaire n'est pas nouveau: à siècle dernier, les premières bibliothèques publiques étaient à peine apparues aux États-Unis qu'on leur confiait déjà une mission éducative. Certaines expériences entreprises au Québec furent sans lendemain parce qu'il s'agissait beaucoup plus de juxtaposition que d'intégration des services. L'idée de l'intégration des deux réseaux, reprise récemment par le gouvernement du Québec, se heurte à deux réalités: la très grande différence des milieux à desservir (scolaire et public) et l'évolution même des bibliothèques publiques qui deviendront sans doute davantage des services d'information que des agents d'éducation.

The concept of the integration of public and school libraries is not new: in the last century the first public libraries had barely started in the United States and already they were being given a role in education. Certain experiences undertaken in Québec were unsuccessfull because they were more a juxtaposition than an integration of services. The idea of the integration of the two networks, recently revived by the Québec government, runs into two difficulties: the very great difference of the two clienteles to be served (school and public), and the evolution of the public libraries which will, without a doubt, become much more information rather than education oriented.

La integración de las bibliotecas públicas y escolares no es un concepto nuevo: apenas habian sido creadas las primeras bibliotecas públicas en los Estados Unidos cuando se les confió una misión educativa. En Québec, hubo algunas experiencias que no tuvieron ningún resultado, porque se trataba de yuxtaposición más que de integración de los servicios. La idea de integrar las dos redes, que el gobierno de Québec acaba de reconsiderar, se enfrenta con dos realidades: la diferencia muy grande entre los grupos de lectores (escuelas y público) y el desarrollo mismo de las bibliotecas públicas que llegarán a ser probablemente servicios de información más que agentes de educación.

La récente récession économique a certes lourdement touché les bibliothèques: les administrateurs ont dû effectuer des prouesses pour réussir à maintenir le fonctionnement des bibliothèques à un niveau acceptable de qualité, avec un personnel réduit et de la documentation à un prix inflationniste. Par contre, cette récession a provoqué chez ces mêmes administrateurs le souci d'une meilleure utilisation et d'une plus grande rationalisation des postes budgétaires, ainsi que l'établissement de politiques et de priorités.
C'est dans cette optique que les hommes politiques, les administrateurs et les contribuables ont exigé une saine administration et un rendement maximum des fonds publics.

Ainsi, assiste-t-on régulièrement à la reprise du débat sur le dédoublement ou la concurrence des bibliothèques publiques et des bibliothèques scolaires à l'intérieur d'une même communauté. 


\section{Origine}

Cette question des bibliothèques à double allégeance est, en fait, aussi vieille que le concept de bibliothèque publique, puisque l'une et l'autre sont interreliés.

Les premières bibliothèques publiques ont vu le jour aux États-Unis au début du dix-neuvième siècle. On connaissait certes déjà diverses formules, notamment les clubs de lecture de Benjamin Franklin, mais il s'agissait de bibliothèques dont il fallait être membres pour y avoir accès. Ce nouveau type de bibliothèque apparaît donc en Nouvelle-Angleterre vers $1837-1840$ et doit répondre à des impératifs éducationnels.

Les préoccupations majeures des intellectuels Everett et Ticknor, lors de la création du Boston Athenaeum, sont de fournir à l'individu les possibilités de s'auto-éduquer, de se former.

Quant aux "Mechanics Institute» qui apparaîtront un peu plus tard, en plus du rôle éducatif, on y ajoutera un objectif de formation de l'ouvrier, de même que l'acquisition de certaines vertus, comme la tempérance.

La mission éducative est donc partie intégrante du concept de la bibliothèque publique; elle s'est toutefois modifiée au fil des années.

En Ontario, dès 1850, Egerton Ryerson, alors surintendant de l'Éducation, faisait la promotion de la bibliothèque publique logée dans les écoles.

"Ryerson regarded the provision of public library service as an essential part of his educational programme (...) Ryerson's public libraries were integrated with education and in many cases placed within the schools so that their facilities could be shared by the whole community, including the students and the teachers of the school. " ${ }^{1}$

1. Gordon Th. Stubbs, The role of Egerton Ryerson in the Development of Public Library Service in Ontario, Ottawa, CLA, 1966, p. 1
Lorsque J.-P. L'Allier, dans son Livre vert intitulé Pour l'évolution de la politique culturelle ${ }^{2}$, avance l'idée des bibliothèques à double allégeance ou la question d'une meilleure utilisation des équipements publics, il reprend un problème plus que centenaire. Selon lui, les études faites sur le sujet sont «trop sectorielles et non concluantes» et cette question de bibliothèque scolaire-publique constitue un "problème réel et un faux débat».

\section{Le débat}

En parcourant la documentation sur le sujet depuis 1960 , on se rend vite compte que le débat a peu évolué.

La principale recherche scientifique, du moins la première, est l'enquête menée par Ruth White auprès des bibliothécaires américains. Elle distingue les deux réseaux de bibliothèques selon différentes variables: les objectifs poursuivis par chacune des bibliothèques, l'administration relevant de juridictions différentes, l'aménagement physique des lieux, la localisation géographique, les clientèles respectives, l'approche psychologique face à ces clientèles, les collections différentes, les exigences de I'horaire et l'économie réalisée. Elle situe le problème:

"Although both public libraries and schools are educational institutions with similar aims, the approach, specific purposes, and methods of operation are different. The public library has an obligation to serve all the people which means all ages; it is characterized by the voluntary nature of its use and its informality (...) the school library is not a separate and distinct institution but is an essential part of the school. ${ }^{3}$

La documentation abonde en exemples où, sous la pression de politiciens ou d'ad-

2. Jean Paul L'Allier, Pour l'évolution de la politique culturelle, Québec, Ministère des Affaires culturelles, 1976, p. 166.

3. Ruth M. White, The School-housed Public Library - A Survey, Chicago, ALA, 1963, p. 1. 
ministrateurs, on a voulu fusionner les deux services de bibliothèque sous le prétexte de réaliser des économies. Ce qui distingue la majorité des articles sur le sujet (plus de 300 entre 1965 et 1976), c'est le caractère local de l'expérience ou l'aspect polémique et récapitulatif de la question.

"To establish a public library in a school environment is to do a disservice to the school, the community and the library staffs. Because of its location in the school, the staff, book collections and service will be oriented to the school curriculum. At best, service to the general public will be marginal. $n^{4}$

Le débat sur les bibliothèques à double allégeance a surtout porté sur des modalités de coopération et rarement sur l'intégration complète d'une bibliothèque à l'autre réseau.

\section{Les expériences québécoises}

Le Québec ne se situe pas en marge de ce courant et plusieurs municipalités ont voulu tenter l'expérience de la cohabitation des deux réseaux de bibliothèques.

La seule réussite en ce sens fut celle de Marieville (dans le comté de Rouville) où la municipalité a bénéficié d'un legs d'un franco-américain qui a doté la ville d'une bibliothèque publique. Un bibliothécaire professionnel avait la responsabilité de ce centre qui devait aussi desservir le milieu scolaire. Toutefois, la construction d'une école polyvalente a mis fin à l'expérience et chacun a réintégré ses fonctions propres.

Pour connaître d'autres expériences québécoises, nous vous référons à l'ouvrage intitulé Profil d'un centre documentaire multimédia $^{5}$ qui en énumère quelquesunes.

4. Ida Reddy, "The school-housed public library - an evaluation». Ontario Library Review, vol. 52, no. 2 (June 1968), 83.

5. J. Klara Szpakowska, Profil d'un centre documentaire multimédia, Montréal, École de bibliothéconomie, 1975 , p. 344-346.
Ces tentatives, selon nous, sont fort discutables et rejoignent celles faites aux États-Unis et au Canada: elles sont très particulières et ne font pas évoluer le débat puisqu'il s'agit de juxtaposition et non d'intégration des services. Sous prétexte de vouloir servir différentes catégories d'usagers en réalisant des économies, on offre un service à rabais à une partie de la population.

L'éducation permanente relevant du ministère de l'Éducation du Québec, on pourrait s'attendre à ce que les bibliothèques scolaires jouent un rôle de complémentarité dans leur formation. À cause de certains facteurs, tels les horaires, le personnel, les différentes juridictions, les contraintes syndicales', les bibliothèques scolaires n'ont pas joué leur rôle et ont laissé au secteur public la responsabilité de la formation documentaire de la population adulte.

Face à une telle situation, le Comité consultatif du livre lui-même, dans ses recommandations et commentaires sur le Livre vert du ministre L'Allier, réoriente les modalités de coopération:

"Les bibliothécaires, se basant sur des expériences réalisées au Québec et à l'étranger, ne croient plus depuis longtemps à la rentabilité culturelle de l'approche dite de fusion, même dans le cadre d'une philosophie de l'éducation permanente. Les coûts d'opération, en dehors des heures normales de travail dans une école, se sont avérés hors de proportion avec le service que l'on pourrait donner les soirs et les fins de semaine. II est préférable de s'orienter vers une complémentarité de services à donner dans ces deux types de bibliothèques, puisqu'ils répondent à des besoins différents; sans compter que les bibliothèques scolaires sont à plusieurs

6. F. Morin et M. Boivin, L'utilisation des laboratoires, bibliothèques et moyens audio-visuels en éducation des adultes, Québec, Ministère de l'Éducation, Direction générale de l'éducation des adultes, 1973, $150 \mathrm{p}$. 
égards plus déficientes encore que les bibliothèques publiques. ${ }^{7}$

\section{Nouveaux besoins... nouvelles formules}

Pendant que les bibliothécaires ou les politiciens dissertaient sur le sujet, les mutations de la société engendraient de nouveaux besoins.

Ainsi, on a reconnu le fait que d'autres organismes extérieurs à l'école peuvent poursuivre une mission éducative, que l'on cherche même à éduquer en dehors du système scolaire, que l'on utilise de plus en plus la technologie comme moyen pédagogique, que la population est de plus en plus impliquée dans un processus d'éducation continue ou récurrente, que diverses formules pédagogiques ont été tentées; l'école n'étant plus le seul centre d'apprentissage, il a donc fallu que les bibliothèques s'adaptent et répondent à de nouveaux besoins.

Les bibliothécaires américains ont alors cherché une formule qui transcenderait toutes les distinctions et offrirait ou mettrait à la disposition de la collectivité toutes les ressources documentaires et d'information disponibles. Cette formule a été appelée aux États-Unis le "Total Community Library Service».

Ce concept a toutefois ses exigences. Cette bibliothèque communautaire ainsi constituée est plus qu'un centre éducatif supplémentaire; elle devient une extension du milieu et du foyer, tous les médias doivent y être disponibles et l'on doit initier les usagers à cette technologie. Une telle bibliothèque doit trouver les moyens de rejoindre le milieu et même d'aménager ses locaux près de ce dernier de façon à rejoindre les usagers actuels et potentiels. Ceci implique un nouveau rôle de la bibliothèque en termes de ressources et de services; à l'occasion, on invite même le milieu à définir lui-même ses besoıns ou à formuler des programmes éducatifs qui répondent à ses attentes.

II existe une variante de cette bibliothèque communautaire et elle prend diverses formes: le "Neighborhood Information Center" aux États-Unis et le "Citizens Advising Bureau" ( $C A B)$ en Angleterre.

Ce qui caractérise ces diverses formules, c'est la préoccupation majeure de fournir de l'information à toute la population et de délaisser les secteurs éducatif, récréatif et culturel. En fait, il s'agit de fournir aux citoyens défavorisés l'information sur divers programmes sociaux et de les orienter parmi cette jungle de l'information.

Comme les bibliothèques continuaient à jouer des rôles plutôt traditionnels et établissaient entre elles des mécanismes de coopération, surtout dans les secteurs de la référence, de la bibliographie et des services techniques, ces centres d'information communautaire se sont impliqués dans leur milieu et ont justement tenté de mobiliser la communauté afin qu'elle formule des programmes qui correspondent à ses besoins.

Quelques bibliothèques publiques se sont dotées d'un tel service que l'on nomme "service d'information et de référence" (Information and Referral Services). Au Québec, de tels services existent. Ils sont fournis, non par des bibliothèques, mais par des agences ou organismes gouvernementaux et privés. Que l'on pense au Centre de référence du grand Montréal ${ }^{8}$ qui fournit de l'information sur les programmes sociaux et éducatifs s'adressant à la population, ou encore aux bureaux de Communication-Québec implantés dans toutes les régions du Québec et qui ont pour but d'informer la population sur les services gouvernementaux. Les bibliothèques publiques, par le biais des publications officielles et gouvernementales, pourraient facilement jouer ce rôle.

Pour remplir cette fonction et devenir un véritable centre d'information, la bi-

8. S. Ledoux et F. Bourbeau, "Rôle social et information communautaire». Argus, vol. 6, no 3-4 (maiaoût 1977), 57-58. 
bliothèque et le bibliothécaire devront concevoir l'information de façon globale, c'està-dire qu'ils ne devront plus se préoccuper du support mais mettre davantage l'accent sur le contenu.

\section{Conclusion}

Les bibliothèques publiques, créées à l'origine pour répondre à des besoins d'éducation, ont peu à peu associé leur rôle au loisir et à la culture et se sont finalement distinguées du circuit scolaire.
Bien que séculaire, le débat sur l'intégration complète des deux réseaux, pour de prétendues écunomies, a peu évolué, ne s'appuie que sur des expériences locales, pleines de particularités, et se déroule sur un ton polémique.

Le rôle d'information n'étant pas complètement assumé par certaines bibliothèques et les besoins n'étant pas satisfaits, on a vu apparaître diverses formules de services qui ont mis l'accent sur les besoins d'information de la collectivité.

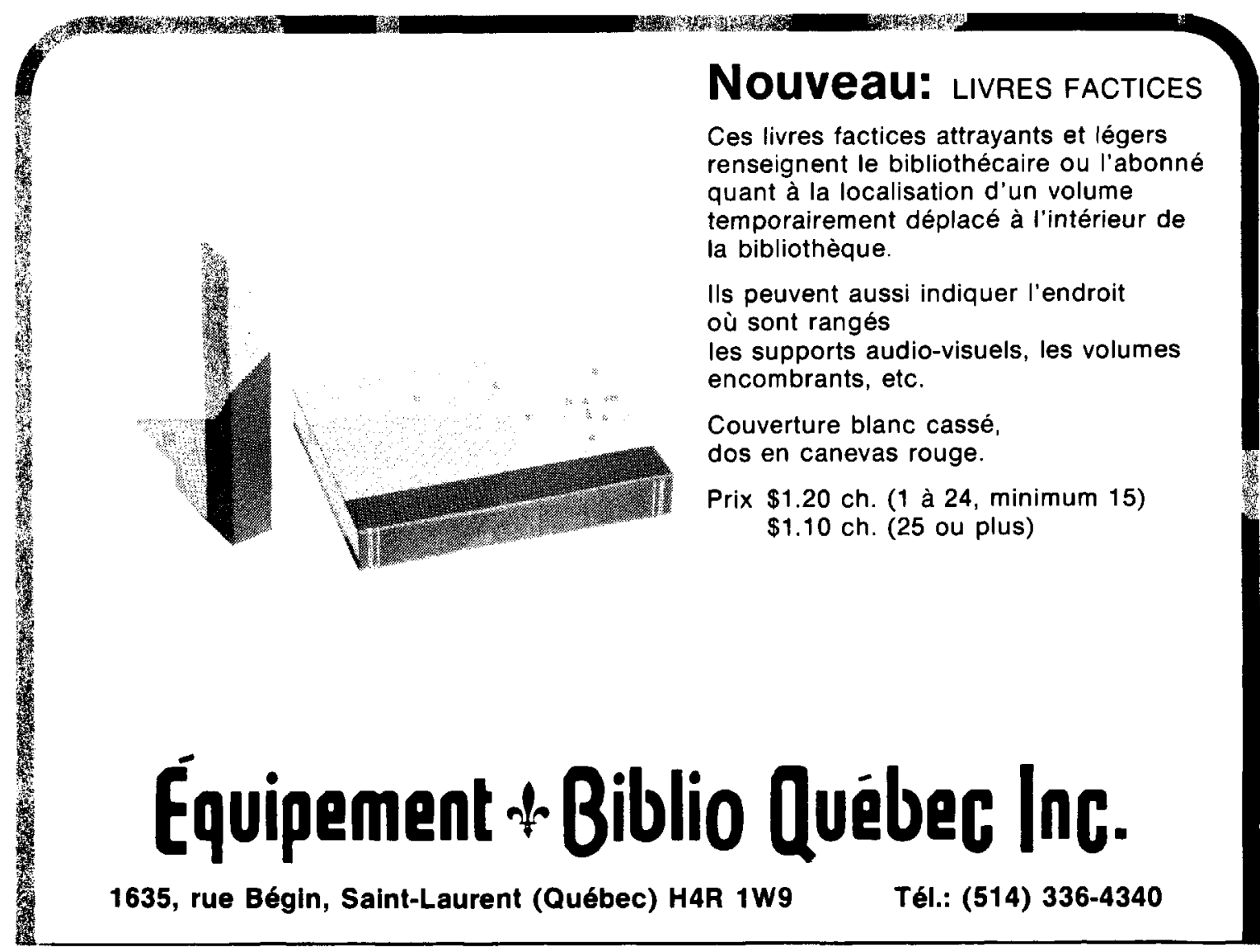

